\title{
Efficient Online Evaluation of Big Data Stream Classifiers
}

\author{
Albert Bifet \\ Noah's Ark Lab \\ HUAWEI \\ Hong Kong \\ bifet.albert@huawei.com \\ Jesse Read \\ HIIT \\ Aalto University \\ Helsinki, Finland \\ jesse.read@aalto.fi
}

\author{
Gianmarco \\ De Francisci Morales \\ Aalto University \\ Helsinki, Finland \\ gdfm@acm.org \\ Geoff Holmes, \\ Bernhard Pfahringer \\ University of Waikato \\ Hamilton, New Zealand \\ \{geoff,bernhard\}@waikato.ac.nz
}

\begin{abstract}
The evaluation of classifiers in data streams is fundamental so that poorly-performing models can be identified, and either improved or replaced by better-performing models. This is an increasingly relevant and important task as stream data is generated from more sources, in real-time, in large quantities, and is now considered the largest source of big data. Both researchers and practitioners need to be able to effectively evaluate the performance of the methods they employ. However, there are major challenges for evaluation in a stream. Instances arriving in a data stream are usually timedependent, and the underlying concept that they represent may evolve over time. Furthermore, the massive quantity of data also tends to exacerbate issues such as class imbalance. Current frameworks for evaluating streaming and online algorithms are able to give predictions in real-time, but as they use a prequential setting, they build only one model, and are thus not able to compute the statistical significance of results in real-time. In this paper we propose a new evaluation methodology for big data streams. This methodology addresses unbalanced data streams, data where change occurs on different time scales, and the question of how to split the data between training and testing, over multiple models.
\end{abstract}

\section{Categories and Subject Descriptors}

H.2.8 [Database Management]: Database Applications - Data Mining

\section{Keywords}

Data Streams, Evaluation, Online Learning, Classification

Permission to make digital or hard copies of all or part of this work for personal or classroom use is granted without fee provided that copies are not made or distributed for profit or commercial advantage and that copies bear this notice and the full citation on the first page. Copyrights for components of this work owned by others than the author(s) must be honored. Abstracting with credit is permitted. To copy otherwise, or republish, to post on servers or to redistribute to lists, requires prior specific permission and/or a fee. Request permissions from Permissions@ acm.org.

KDD '15, August 10-13, 2015, Sydney, NSW, Australia.

Copyright is held by the owner/author(s). Publication rights licensed to ACM. ACM 978-1-4503-3664-2/15/08\$15.00.

DOI: http://dx.doi.org/10.1145/2783258.2783372.

\section{INTRODUCTION}

Data is flowing into every area of our life, both professional and personal. There is no consensus on the definition of big data, however a popular definition describes it as data whose characteristics put it beyond the ability of typical tools to capture, manage, and analyze, due to time and memory constraints 21]. This need has spurred a considerable amount of research in data mining and machine learning, aimed at extracting information and knowledge from big data.

In this paper, we focus on the evaluation of classifiers in a big data setting such as the one provided by APACHE $\mathrm{SAMOA}^{1}$ 9] i.e., classifying evolving data streams in a distributed fashion. When the classification algorithm runs on top of a distributed system, we can leverage its processing power to train multiple classifiers and provide statistical significance to the evaluation procedure. There is no previous work that describes an evaluation methodology that gives statistically significant results in streaming classification, going beyond the standard prequential setting which builds only one model for the evaluation. Providing statistical significance is of paramount importance in order to ensure that evaluation results are valid and not misleading.

Evaluation is a delicate task that has generated numerous controversies 14, 18. As mentioned by Japkowicz and Shah [18, having a standard evaluation approach can be linked to the desire of having an "acceptable" scientific practice in the field. Unfortunately, researchers often achieve such acceptability by taking shortcuts. The problem with this practice is that the comparisons of algorithms' performance, although appearing acceptable, are frequently invalid. In this paper we highlight the importance of a proper evaluation methodology for streaming classifiers, and contribute to this discussion by presenting some insightful results. From these, we develop and present some effective new proposals.

To motivate the need for this discussion, we present three striking cases of misleading evaluation (details in $\$ 4$ and \$5):

- by comparing two classifiers applied to data streams, we show that one of them is statistically significantly better than the other by using McNemar's test. However, the two classifiers are actually two instances of the same algorithm, randomized ensembles of decision trees, each seeded with a different number. A test such as McNe-

\footnotetext{
http://samoa-project.net
} 
mar's, which works properly for small datasets, is misleading when used with larger ones. Nevertheless, this test is commonly used for data stream classification [24;

- splitting data into several disjoint datasets for training seems reasonable for large datasets [11. Unfortunately, this type of partitioning leads to an evaluation procedure that cannot distinguish between classifiers that ought to perform differently by design;

- a simple majority class classifier that keeps the majority class of a sliding window may have positive $\kappa$ statistic and positive harmonic mean accuracy for some periods.

Learning from evolving data streams is quite different from learning from traditional batch data. Standard crossvalidation techniques do not apply, due to the fact that data instances can be strongly time-dependent, and classifiers also evolve along with the data. It is no use training a classifier on a large number of data instances, and then evaluating it on some following data (analogous to a static train/test split) without letting classifiers evolve with the new data. In a big data stream scenario, the instances in the test set could be millions of instances away from the ones used to train the model. A classifier may be built using some features as the most relevant, but when the data changes, the classifier has to adapt and choose new relevant features. It is therefore useful to have a statistically valid way to measure the performance of classifiers at any time. Current frameworks for data streams such as $\mathrm{MOA}^{2}$ [3], VFML [16], and Vowpal Wabbit 20 provide only single fold prequential evaluation. Therefore, they fall short of a statistically sound evaluation setting.

In this work we identify a set of currently popular approaches that are problematic, highlight the issues arising from the big data streaming setting, and finally propose some solutions. Specifically, we tackle the following issues:

I1. Validation methodology. Prequential evaluation, the most commonly used evaluation in stream learning, uses examples first to test and then to train a single model. This method is unable to provide statistical significance when comparing classifiers. On the other hand, the most common error estimation technique for the batch setting, cross-validation, is not directly applicable in streaming.

I2. Statistical testing. McNemar's test, which has been proposed as one of the best tests for streaming, is misleading.

I3. Unbalanced measure. Common measures of performance such as F1 and Accuracy are biased toward one class.

I4. Forgetting mechanism. The most popular approaches, sliding window and exponential forgetting, are both parametric with difficult to choose parameters. In addition, they allow a mix of distributions in the input window.

To address these issues we propose: a new prequential bootstrap validation to address I1; to use the Sign test or

\footnotetext{
2 http://moa.cms.waikato.ac.nz

3 http://www.cs.washington . edu/dm/vfml

4 http://hunch.net/ vw
}

the Wilcoxon signed-rank test to address I2; a new measure for accuracy performance, $\kappa_{m}$ statistic to address I3, and a new forgetting mechanism for prequential evaluation based on ADWIN to address I4.

This paper is structured as follows. We present some related work in Section 2, distributed validation methodologies in Section 3 statistical tests in Section 4 evaluation performance measures in Section 5 and a real-time prequential measure in Section 6. Finally, Section 7 presents some concluding remarks.

\section{RELATED WORK}

The most important reference in evaluating data streams is by Gama et al. [13. The paper mainly discusses static streams, and how to extend the static streaming prequential evaluation to evolving data streams using a sliding window or a fading factor.

Dietterich 11 reviewed five approximate statistical tests for determining whether one learning method out-performs another on a particular classification task. These tests were compared experimentally to check the type I and type II errors on the differences. The main conclusion was that $5 \times 2$ cross validation test was recommended, due to it being slightly more powerful than McNemar's test.

Bouckaert [6] recommends using the 10x10 cross validation test where all individual accuracies are used to estimate the mean and variance and with 10 degrees of freedom for binary data. It has the same properties as other calibrated repeated k-fold cross validation tests, and he showed that it empirically outperforms $5 \times 2$ cross validation, (corrected) resampling and k-fold cross validation on power and replicability.

Demsar [10] reviewed statistical tests for comparing more than two algorithms on multiple datasets. One of his proposals is to use the Friedman test with a corresponding post-hoc test, that we discuss in more detail in Section 4

Shah 25. proposed a new agreement statistic that is a generalization of the Cohen's kappa statistic to the case of multiclass classification by a fixed group of experts. The proposed generalization reduces to the classical version of Cohen's kappa statistic in the case of binary classification by two experts. Its main advantage is that it yields tighter agreement assessments, due to its accounting for expert specific biases and correlations.

More generally, the recent book by Japkowicz and Shah 18 is a complete and updated reference for classification evaluation.

\section{METHODS FOR VALIDATION}

The validation procedure for a learning algorithm determines which examples are used for training, and which are used for testing the learned model.

In traditional batch learning the problem of limited availability of data is overcome by analyzing and averaging multiple models produced with different random arrangements of training and test data. In the stream setting the problem of (effectively) unlimited data poses different challenges.

In the literature there are two main approaches to evaluate data streams:

- prequential evaluation, averaging ten experiments when learners or streams are randomly generated, and when it is possible to vary their random seeds; otherwise by 


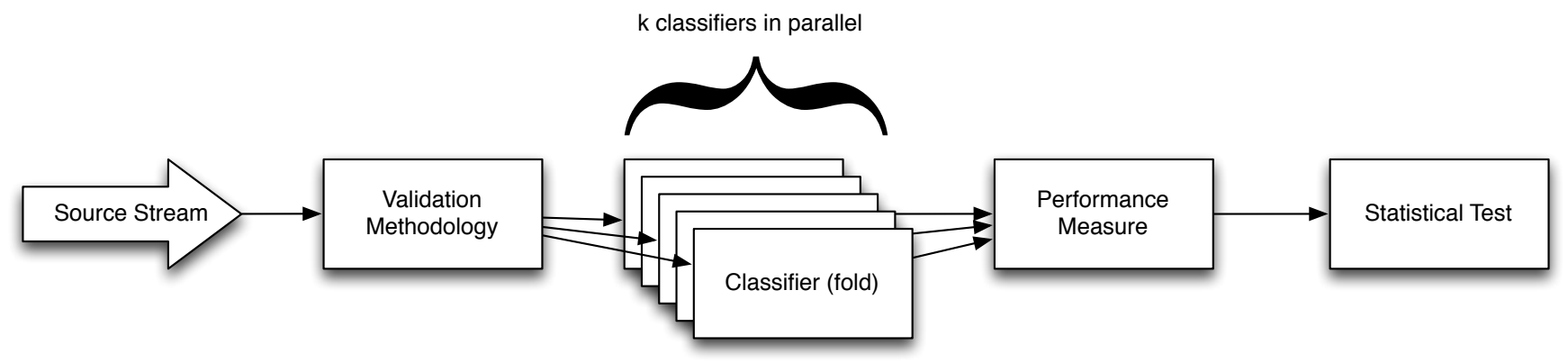

Figure 1: Block diagram of the proposed evaluation pipeline for big data stream classifiers.

using only one experiment with real data datasets and non randomized classifiers;

- standard 10-fold cross-validation, to compare with other batch methods.

The former cannot be used to obtain statistical significance of results when using real datasets with any nonrandomized classifier. Furthermore, this strategy is not amenabl to parallelization. The latter treats each fold of the stream independently, and therefore may miss concept drift occuring in the data stream.

To overcome these problems, we discuss the following strategies. Assume we have $k$ different instances of the classifier we want to evaluate running in parallel. The classifier does not need to be randomized. Each time a new example arrives, it is used in one of the following ways:

- $k$-fold distributed cross-validation: each example is used for testing in one classifier selected randomly, and used for training by all the others;

- $k$-fold distributed split-validation: each example is used for training in one classifier selected randomly, and for testing in the other classifiers;

- $k$-fold distributed bootstrap validation: each example is used for training in each classifier according to a weight from a Poisson(1) distribution. This results in each example being used for training in approximately two thirds of the classifiers, with a separate weight in each classifier, and for testing in the rest.

The first approach is an adaptation of cross-validation to the distributed streaming setting. It makes maximum use of the available data at the cost of high redundancy of work.

The split-validation approach has the advantage of creating totally independent classifiers, as they are trained on disjoint parts of the stream. However, this approach potentially underutilizes available data.

The last approach simulates drawing random samples with replacement from the original stream. This approach is also used in online bagging [23, 22 .

All three strategies have a corresponding prequential version, where training examples are first used for testing.

A high level block diagram of the whole evaluation pipeline is shown in Figure 1

\subsection{Streaming Setting}

In the streaming setting, classifiers and streams can evolve over time. As a result, the performance measures of these classifiers can also evolve over time. Given this dynamic na- ture, it is interesting to be able to evaluate the performance of several classifiers online.

For simplicity, let us assume a binary classification problem. The ideal setting for evaluation when data is abundant is the following: let $X$ be the instance space, let $\mathcal{D}_{t}$ be a distribution over $X$ and time, let $f_{t}$ be a target function $f_{t}: X \rightarrow[0,1]$, and let $c_{t}$ be a model evolving over time, so that it can be different at each time instant $t$. A classifier $C$ is a learning algorithm that produces a hypothesis $c_{t}: X \rightarrow[0,1]$.

We are interested in obtaining its error $e_{c(t)}(x)=\mid c_{t}(x)-$ $f_{t}(x) \mid$, which also depends on time. The true error is $\bar{e}_{c(t)}=$ $E_{x \in \mathcal{D}}\left[e_{c(t)}(x)\right]$. Let the error discrepancy be the difference between the true and estimated error. In $k$-fold evaluation the estimated error is the average over all fold estimates.

\subsection{Theoretical Insights}

Let us mention here two important theoretical results.

TheOREM 1. (Test Set Lower Bound) [19] For all classifiers $c, m$ test instances, and for all $\delta \in(0,1]$, the following holds:

$$
\operatorname{Pr}\left(\mid \text { true error - est. error } \mid \leq \sqrt{\frac{\ln (2 / \delta)}{2 m}}\right) \geq 1-\delta
$$

In other words, with the same high probability, having more instances will reduce the discrepancy between the true error and the estimated error, which, in our case, suggests that a prequential strategy (using the instances for testing before training) improves the estimation of the true error.

Following the approach by Blum et al. [5], the second result is that the discrepancy between true error and estimated error is reduced using a $k$-fold strategy.

THEOREM 2. $\forall q \geq 1, E\left[\mid\right.$ true error - est. error $\left.\left.\right|^{q}\right]$ is no larger for the prequential $k$-fold strategy than for a prequential evaluation strategy, i.e.,

$$
\begin{gathered}
E\left[\mid \text { true error }_{k}-\text { estimated } \text { error }\left._{k}\right|^{q}\right] \leq \\
E\left[\mid \text { true error }_{1}-\text { estimated } \text { error }\left._{1}\right|^{q}\right]
\end{gathered}
$$

In other words, using $k$-fold prequential evaluation is better than using only prequential evaluation, and gives us theoretical ground for addressing issue I1. Given the interdependence between validation methodology and statistical tests, we defer experimental evaluation to Section 4 
Table 1: Comparison of two classifiers with Sign test and Wilcoxon's signed-rank test.

\begin{tabular}{cccc}
\hline Class. A & Class. B & Diff & Rank \\
\hline 77.98 & 77.91 & 0.07 & 4 \\
72.26 & 72.27 & -0.01 & 1 \\
76.95 & 76.97 & -0.02 & 2 \\
77.94 & 76.57 & 1.37 & 7 \\
72.23 & 71.63 & 0.60 & 5 \\
76.90 & 75.48 & 1.42 & 8 \\
77.93 & 75.75 & 2.18 & 9 \\
72.37 & 71.33 & 1.04 & 6 \\
76.93 & 74.54 & 2.39 & 10 \\
77.97 & 77.94 & 0.03 & 3 \\
\hline
\end{tabular}

\section{STATISTICAL TESTS FOR COMPARING CLASSIFIERS}

The three most used statistical tests for comparing two classifiers, are the following [18]:

- McNemar. This test is non-parametric. It uses two variables: the number of examples misclassified by the first classifier and correctly classified by the second $a$, and the number of examples classified the opposite way $b$. Hence, it can be used even with a single-fold validation strategy. The McNemar statistic $(M)$ is computed as $M=\operatorname{sign}(a-b) \times(a-b)^{2} /(a+b)$. The statistic follows the $\chi^{2}$ distribution under the null hypothesis that the two classifiers perform equally well.

- Sign test. Is also non-parametric but uses the results at each fold as a trial. As such, it cannot be used with a standard prequential strategy. It computes how many times the first classifier outperforms the second classifier, and how many times the opposite happens. The null hypothesis corresponds to the two classifiers performing equally, and holds if the number of wins follows a binomial distribution. If one of the classifiers performs better on at least $w_{\alpha}$ folds, then it is considered statistically significantly better at the $\alpha$ significance level, where $w_{\alpha}$ is the critical value for the test at the $\alpha$ confidence level.

- Wilcoxon's signed-rank test. Is also non-parametric, and uses the results at each fold as a trial. For each trial it computes the difference in performance of the two classifiers. After ranking the absolute values of these differences, it computes the sum of ranks where the differences are positive, and the sum of ranks where the differences are negative. The minimum value of these two sums is then compared to a critical value $V_{\alpha}$. If this minimum value is lower, the null hypothesis that the performance of the two classifiers is the same can be rejected at the $\alpha$ confidence level.

Consider the following example: two classifiers have 10 performance measures, one for each fold, as shown in Table 1 Classifier A outperforms classifier B in eight folds. Using the sign-test, given that for $\alpha=.05$ we have $w_{\alpha}=8$, we can reject the null hypothesis that the two classifiers perform similarly at the $\alpha=.05$ confidence level. To apply the Wilcoxon's signed-rank test, first we rank the absolute values of the differences between the two classifiers' performances. Then we compute the sum of ranks where the differences are positive (53), and the sum of ranks where the differences are negative (3). We compare the minimum of these two sums (3) to a critical value $V_{\alpha}=8$ at the $\alpha=.05$ confidence level. As 3 is lower than 8 , we reject the null hypothesis that the the two classifiers perform equally well.

This procedure can be extended to compare multiple classifiers. Demsar 10 proposes to use the Friedman test with a corresponding post-hoc test, such as the Nemenyi test. Let $r_{i}^{j}$ be the rank of the $j$-th of $k$ classifiers on the $i$-th of $N$ datasets. The average rank of the $j$-th classifier is $R_{j}=1 / n \sum_{i} r_{i}^{j}$. The Nemenyi test is the following: two classifiers are performing differently if the corresponding average ranks differ by at least the critical difference

$$
C D=q_{\alpha} \sqrt{\frac{k(k+1)}{6 N}}
$$

where $k$ is the number of classifiers, $N$ is the number of datasets, and critical values $q_{\alpha}$ are based on the Studentized range statistic divided by $\sqrt{2}$.

\subsection{Type I and II Error Evaluation}

A Type I error or false positive is the incorrect rejection of a true null hypothesis, in our case, that two classifiers have the same accuracy. Our experimental setting is the following: we have a data stream, and we use it as input to classifiers that have the same accuracy by design since they are built using the same algorithm. Then, we check which evaluation methodology is better at avoiding the detection of a difference between statistically equally-performing classifiers, i.e., which method has a lower type I error.

We run an experiment following the methodology presented by Dietterich [11], and build randomized classifiers with different seeds. We use two different models trained with random forests [4, and compare them with the statistical tests discussed in this Section.

A Type II error or false negative is the failure to reject a false null hypothesis, in our case, to detect that two classifiers have different accuracy. For detecting this type of error, we perform the following experiment. We have a data stream that we use to feed a set of classifiers. These classifiers have different accuracy performance by design. We build this set of classifiers by using a base classifier and applying a noise filter. The noise filter changes the class label of each example prediction of the classifier with probability $p_{\text {noise }}$. For a $c$ class problem, if the accuracy of the original classifier is $p_{0}$, the filtered classifier has an accuracy of:

$$
p=p_{0} \times\left(1-p_{\text {noise }}\right)+\left(1-p_{0}\right) \times p_{\text {noise }} / c
$$

since $p$ will be $p_{0}$ minus the correctly predicted examples that have switched their label $\left(p_{0} \times p_{\text {noise }}\right)$, plus the incorrect predicted examples that due to the switch of the class label, are now correct $\left(1-p_{0}\right) \times p_{\text {noise }} / c$. Given that

$$
\frac{\Delta p}{p_{\text {noise }}}=\frac{p_{0}-p}{p_{\text {noise }}}=\frac{(c+1) p_{0}-1}{c}
$$

$p$ will be lower than $p_{0}$ when $p_{0}>1 /(c+1)$.

Experimental Setting. We compare 10-fold distributed evaluation with the non prequential and prequential versions, and with split-validation, cross-validation, and bootstrapping over 50 runs at the $\alpha=0.05$ confidence level.

We use synthetic and real datasets. Synthetic data has several advantages: it is easier to reproduce and there is little cost in terms of storage and transmission. We use the data 
Table 2: Average fraction of null hypothesis rejection for different combinations of validation procedures and statistical tests, aggregated over all datasets. The first column group concerns Type I errors, and the other two column groups concern Type II errors.

\begin{tabular}{|c|c|c|c|c|c|c|c|c|c|}
\hline & \multicolumn{3}{|c|}{ No change } & \multicolumn{3}{|c|}{ Change $p_{\text {noise }}=0.05$} & \multicolumn{3}{|c|}{ Change $p_{\text {noise }}=0.10$} \\
\hline & bootstrap & $\mathrm{cv}$ & split & bootstrap & $\mathrm{cv}$ & split & bootstrap & $\mathrm{cv}$ & split \\
\hline McNemar non-prequential & 0.71 & 0.52 & 0.66 & 0.86 & 0.80 & 0.73 & 0.88 & 0.84 & 0.75 \\
\hline McNemar prequential & 0.77 & 0.80 & 0.42 & 0.88 & 0.94 & 0.56 & 0.90 & 0.95 & 0.58 \\
\hline Sign test non-prequentiall & 0.11 & 0.11 & 0.10 & 0.77 & 0.82 & 0.44 & 0.82 & 0.88 & 0.50 \\
\hline Sign test prequential & 0.12 & 0.12 & 0.09 & 0.77 & 0.83 & 0.44 & 0.82 & 0.89 & 0.50 \\
\hline Wilcoxon non-prequential & 0.11 & 0.11 & 0.14 & 0.79 & 0.84 & 0.51 & 0.83 & 0.90 & 0.55 \\
\hline Wilcoxon prequential & 0.11 & 0.10 & 0.19 & 0.80 & 0.84 & 0.54 & 0.83 & 0.91 & 0.59 \\
\hline Avg. time non-prequential ( $\mathrm{s}$ & 883 & 1105 & 415 & 877 & 1121 & 422 & 900 & 1148 & 421 \\
\hline Avg. time prequential (s) & 813 & 1202 & 109 & 820 & 1214 & 111 & 808 & 1294 & 112 \\
\hline
\end{tabular}

generators with concept drift most commonly found in the literature: SEA Concepts Generator [26], Rotating Hyperplane [17, Random RBF Generator, and LED Generator [7. The UCI machine learning repository [1] contains some realworld benchmark data for evaluating machine learning techniques. We consider two of the largest: Forest Covertype, and Poker-Hand. In addition, we consider the Electricity dataset 15, 12. Experiments can be replicated with the open-source framework APACHE SAMOA 9 .

A summary of results is shown in Table 2. The values show the average fraction of repetitions that detect a difference between the two classifiers, i.e., that reject the null hypothesis. Ideally, the value should be close to zero when the classifiers are the same (first column group), and close to one when the two classifiers are different (second and third column groups). Overall, the best validation procedure is cross-validation, closely followed by bootstrap, while splitvalidation performs the worst. For Type II errors, most statistical tests perform comparably when using a good validation method (cv or bootstrap), i.e., the tests detect the presence of the additional noise. Conversely, for Type I errors there is a large performance gap. McNemar's test is clearly misleading as it detects a non-existent difference at least $42 \%$ of the time, while in fact the two classifiers are built by the same algorithm.

Table 3 shows a breakdown of the results for McNemar's test on each dataset. It is evident that the test has a tendency to overestimate the statistical difference of two classifiers. On the other hand, Table 4 shows the results for Wilcoxon's signed-rank test for all data streams. This test is more discriminative than the Sign test, and the best strategies are still cross-validation and bootstrap. In most cases (with the exception of the SEA generator), Wilcoxon's test has perfect accuracy for Type II errors, and very high accuracy for Type I errors ( $\leq 10 \%$ average error).

A plausible explanation for the difference among the different validation procedures is rooted in the number of examples that each classification algorithm uses to build the model. In 10-fold split validation, the models are built on $10 \%$ of the examples, in 10 -fold cross validation, the models are built on $90 \%$ of the examples, and in bootstrap, the models use $63.2 \%$ of the examples (on average). The experiments show that building the models in each fold with more examples helps to determine whether the classifiers are statistically different, for both Type I and Type II errors.

In terms of efficiency, by looking at the average time of the experiments in Table 2 we conclude that bootstrapping is the most efficient methodology, as it is the one with the best ratio of discriminative power against resources consumed.
There is little difference in the results between the prequential and non-prequential version of the experiments. The prequential version has the advantage that models do not need to receive separate examples for testing, only receiving training examples is enough.

Based on these results, we recommend the prequential $k$ fold distributed bootstrap validation procedure as the most efficient methodology to address issue I1. We also recommend avoiding McNemar's test, and using Wilcoxon's signedrank test to address issue I2.

The main intuition behind the inappropriateness of McNemar's test lies in its use of examples rather than folds as the unit of its statistical treatment. Given that in a stream the number of examples (sample size) is virtually unbounded, the statistical power of the test (its sensitivity) becomes very high, and also susceptible to small differences in performance (magnitude of the effect) caused by random fluctuations. Tests using folds as their statistical unit, such as Wilcoxon's, are thus inherently more robust, as their power is fixed by the configuration, rather than increasing with the number of examples in the stream. Of course, folds are not independent of each other, and the number of folds affects the minimum detectable difference. This statistical dependence is likely the reason behind the residual Type I and Type II errors in our analysis, although further study is needed to characterize its effect.

\section{UNBALANCED PERFORMANCE MEA- SURES}

In real data streams, the number of examples for each class may be evolving and changing. The prequential error is computed based on an accumulated sum of a loss function $L$ between the prediction $y_{t}$ and observed values $\hat{y}_{t}$ :

$$
p_{0}=\sum_{t=1}^{n} L\left(\hat{y}_{t}, y_{t}\right) .
$$

However, the prequential accuracy measure is only appropriate when all classes are approximately balanced [18].

In the following we review standard approaches to evaluation in imbalanced data, then we point out some problems with this methodology, and propose a new one, which we later demonstrate in experiments to be a more accurate gauge of true performance.

\subsection{The Kappa Statistic}

When the data stream is unbalanced, simple strategies that use this fact may have good accuracy. A way to take 
Table 3: Fraction of null hypothesis rejection on each dataset when using McNemar's test. Values for the best performing validation method (closest to zero for Type I errors, closest to one for Type II errors) are shown in bold.

\begin{tabular}{|c|c|c|c|c|c|c|c|c|c|}
\hline & \multicolumn{3}{|c|}{ No change } & \multicolumn{3}{|c|}{ Change $p_{\text {noise }}=0.05$} & \multicolumn{3}{|c|}{ Change $p_{\text {noise }}=0.10$} \\
\hline & bootstrap & $\mathrm{cv}$ & split & bootstrap & $\mathrm{cv}$ & split & bootstrap & $\mathrm{cv}$ & split \\
\hline CovType & 0.86 & 0.64 & 0.98 & 0.97 & 0.98 & 0.98 & 0.98 & 0.99 & 0.99 \\
\hline ELECTRICITY & 0.45 & 0.16 & 0.79 & 0.99 & 0.92 & 0.88 & 1.00 & 1.00 & 0.94 \\
\hline POKER & 0.73 & 0.45 & 0.86 & 0.82 & 0.78 & 0.98 & 0.96 & 0.99 & 1.00 \\
\hline $\operatorname{LED}(50000)$ & 0.92 & 0.85 & 0.96 & 0.96 & 0.92 & 1.00 & 0.99 & 0.96 & 1.00 \\
\hline SEA(50) & 0.67 & 0.35 & 0.88 & 0.69 & 0.39 & 0.89 & 0.70 & 0.43 & 0.89 \\
\hline SEA $(50000)$ & 0.68 & 0.35 & 0.89 & 0.70 & 0.41 & 0.89 & 0.71 & 0.44 & 0.89 \\
\hline $\operatorname{HYP}(10,0.001)$ & 0.61 & 0.32 & 0.61 & 1.00 & 1.00 & 1.00 & 1.00 & 1.00 & 1.00 \\
\hline $\operatorname{HYP}(10,0.0001)$ & 0.61 & 0.40 & 0.83 & 1.00 & 1.00 & 1.00 & 1.00 & 1.00 & 1.00 \\
\hline $\operatorname{RBF}(0,0)$ & 0.89 & 0.80 & 0.36 & 0.98 & 0.98 & 0.39 & 0.99 & 0.99 & 0.40 \\
\hline $\operatorname{RBF}(50,0.001)$ & 0.26 & 0.20 & 0.36 & 0.18 & 0.43 & 0.39 & 0.20 & 0.44 & 0.39 \\
\hline $\operatorname{RBF}(10,0.001)$ & 0.88 & 0.74 & 0.36 & 0.97 & 0.96 & 0.39 & 0.98 & 0.98 & 0.40 \\
\hline $\operatorname{RBF}(50,0.0001)$ & 0.76 & 0.68 & 0.37 & 0.91 & 0.76 & 0.39 & 0.91 & 0.76 & 0.41 \\
\hline $\operatorname{RBF}(10,0.0001)$ & 0.89 & 0.80 & 0.36 & 0.96 & 0.92 & 0.37 & 0.98 & 0.94 & 0.40 \\
\hline Average & 0.71 & 0.52 & 0.66 & 0.86 & 0.80 & 0.73 & 0.88 & 0.84 & 0.75 \\
\hline
\end{tabular}

Table 4: Fraction of null hypothesis rejection on each dataset when using Wilcoxon's Signed-Rank test. Values for the best performing validation method (closest to zero for Type I errors, closest to one for Type II errors) are shown in bold.

\begin{tabular}{|c|c|c|c|c|c|c|c|c|c|}
\hline & \multicolumn{3}{|c|}{ No change } & \multicolumn{3}{|c|}{ Change $p_{\text {noise }}=0.05$} & \multicolumn{3}{|c|}{ Change $p_{\text {noise }}=0.10$} \\
\hline & bootstrap & $\mathrm{cv}$ & split & bootstrap & $\mathrm{cv}$ & split & bootstrap & $\mathrm{cv}$ & split \\
\hline CovType & 0.12 & 0.10 & 0.10 & 0.99 & 1.00 & 0.63 & 1.00 & 1.00 & 0.97 \\
\hline ELECTRICITY & 0.12 & 0.13 & 0.14 & 1.00 & 1.00 & 0.72 & 1.00 & 1.00 & 0.98 \\
\hline POKER & 0.08 & 0.10 & 0.10 & 0.79 & 1.00 & 1.00 & 1.00 & 1.00 & 1.00 \\
\hline LED (50000) & 0.11 & 0.09 & 0.10 & 0.82 & 0.49 & 1.00 & 1.00 & 0.99 & 1.00 \\
\hline SEA(50) & 0.11 & 0.12 & 0.14 & 0.26 & 0.27 & 0.11 & 0.36 & 0.45 & 0.12 \\
\hline SEA $(50000)$ & 0.10 & 0.09 & 0.09 & 0.21 & 0.29 & 0.11 & 0.32 & 0.49 & 0.11 \\
\hline $\operatorname{HYP}(10,0.001)$ & 0.09 & 0.10 & 0.13 & 1.00 & 1.00 & 1.00 & 1.00 & 1.00 & 1.00 \\
\hline $\operatorname{HYP}(10,0.0001)$ & 0.13 & 0.09 & 0.11 & 1.00 & 1.00 & 1.00 & 1.00 & 1.00 & 1.00 \\
\hline $\operatorname{RBF}(0,0)$ & 0.11 & 0.10 & 0.32 & 1.00 & 1.00 & 0.29 & 1.00 & 1.00 & 0.33 \\
\hline $\operatorname{RBF}(50,0.001)$ & 0.14 & 0.10 & 0.30 & 0.32 & 1.00 & 0.33 & 0.13 & 1.00 & 0.29 \\
\hline $\operatorname{RBF}(10,0.001)$ & 0.12 & 0.10 & 0.32 & 1.00 & 1.00 & 0.28 & 1.00 & 1.00 & 0.28 \\
\hline $\operatorname{RBF}(50,0.0001)$ & 0.10 & 0.11 & 0.31 & 1.00 & 0.85 & 0.31 & 0.99 & 0.86 & 0.30 \\
\hline $\operatorname{RBF}(10,0.0001)$ & 0.10 & 0.09 & 0.31 & 1.00 & 1.00 & 0.32 & 1.00 & 1.00 & 0.29 \\
\hline Average & 0.11 & 0.10 & 0.19 & 0.80 & 0.84 & 0.54 & 0.83 & 0.91 & 0.59 \\
\hline
\end{tabular}

this into account, is to normalize $p_{0}$ by using

$$
p_{0}^{\prime}=\frac{p_{0}-\min p}{\max p-\min p}
$$

where $\min p$ and $\max p$ are the minimum and maximum accuracy obtainable in the stream, respectively.

The Kappa statistic $\kappa$ was introduced by Cohen [8]:

$$
\kappa=\frac{p_{0}-p_{c}}{1-p_{c}}
$$

The quantity $p_{0}$ is the classifier's prequential accuracy, and $p_{c}$ is the probability that a chance classifier-one that assigns the same number of examples to each class as the classifier under consideration-makes a correct prediction. If the classifier is always correct then $\kappa=1$. If its predictions are correct as often as those of a chance classifier, then $\kappa=0$.

For example, if a chance classifier accuracy is 0.48 , and prequential accuracy is 0.65 , then $\kappa=\frac{0.65-0.48}{1-0.48}=32.69 \%$ (Table 5).

The efficiency of computing the Kappa statistic is an important reason why it is more appropriate for data streams than a measure such as the area under the ROC curve.

Similarly, the Matthews correlation coefficient (MCC) is a correlation coefficient between the observed and predicted binary classifications defined as: 


\subsection{Harmonic Mean}

Unbalanced measures that use class label accuracies are the arithmetic mean and the geometric mean

$$
\begin{gathered}
A=1 / c \cdot\left(A_{1}+A_{2}+\ldots+A_{c}\right) \\
G=\left(A_{1} \times A_{2} \times \ldots A_{c}\right)^{1 / c},
\end{gathered}
$$

where $A_{i}$ is the prequential accuracy on class $i$ and $c$ is the number of classes, e.g., in a binary classification setting $A_{C+}=T P /(T P+F N)$. Note that the geometric accuracy of the majority vote classifier would be zero, as accuracy on the classes other than the majority would be zero. Perfect classification yields one. If the accuracies of a classifier are balanced across the classes, then the geometric accuracy is equal to standard accuracy.

The harmonic mean is defined as:

$$
H=c /\left(\frac{1}{A_{1}}+\frac{1}{A_{2}}+\ldots \frac{1}{A_{c}}\right) .
$$

The main advantage of the harmonic mean is that, as it is always smaller than the arithmetic mean and the geometric mean $(H \leq G \leq A)$, it tends strongly toward the accuracy of the class with higher error, thus helping to mitigate the impact of large classes and emphasizing the importance of smaller ones.

Note that for a two class problem, $A, G, H$ follow a geometric progression with a common ratio of $G / A \leq 1$, as $G=A \cdot(G / A), H=G \cdot(G / A)$, and $H=A \cdot(G / A)^{2}$.

This measure is inspired by the $F 1$ measure, the harmonic mean between precision and recall:

$$
F 1=\frac{2}{1 / \text { prec. }+1 / \text { recall }}=\frac{2 \cdot T P}{2 T P+F N+F P},
$$

where precision $=\frac{T P}{T P+F P}$ and recall $=\frac{T P}{T P+F N}$.

Note that the F1 measure is not symmetric, as it ignores TN. We can rewrite $F 1$, to include $T N$, as

$$
F 1^{\prime}=\frac{1}{1+1 / 2\left(\frac{F N}{T P}+\frac{F P}{T N}\right)},
$$

which is, in fact, equivalent to the harmonic mean:

$$
\begin{aligned}
H & =\frac{2}{1 / \frac{T P}{T P+F N}+1 / \frac{T N}{T N+F P}} \\
& =\frac{2}{\frac{T P+F N}{T P}+\frac{T N+F P}{T N}}=\frac{1}{1+1 / 2\left(\frac{F N}{T P}+\frac{F P}{T N}\right)} .
\end{aligned}
$$

As computing determinants for dimensions larger than 2 is much more expensive than computing the harmonic mean, the determinant of the confusion matrix $(C M D)$ may be used only for binary classification, and the harmonic mean for multi-class classification.

\subsection{Problems with Kappa Statistic and Har- monic Mean}

Consider the simple confusion matrix shown in Table 5. Class + is predicted correctly 40 out of 100 times, and Classis predicted correctly 25 times. So the accuracy $p_{0}$ is $65 \%$. A random classifier that predicts solely by chance-in the same proportions as the classifier of Table 5-will predict Class + and Class- correctly in $31.50 \%$ and $16.50 \%$ of cases respectively. Hence, it has an accuracy $p_{c}$ of $48 \%$. The $\kappa$ statistic is then $32.69 \%$, MCC is $37.28 \%$, the accuracy for Class + is $57.14 \%$ and for Class- is $83.33 \%$, the arithmetic mean $A$ is $70.24 \%$, the geometric mean $G$ is $69.01 \%$ and the harmonic mean $H$ is $67.80 \%$.

It may seem that as the $\kappa$ statistic is positive $(32.69 \%)$ and harmonic mean is high $(67.80 \%)$, we have a good classifier. However, if we look at the accuracy of a majority class classifier that predicts always Class+, it is $70.0 \%$, since Class+ appears in $70 \%$ and Class- appears in $30 \%$ of examples.

It is commonly, and wrongly, assumed that the $\kappa$ statistic is a measure that compares the accuracy of a classifier with the one of a majority class classifier, and that any majority class classifier will always have $\kappa$ statistic equal to zero. However, as we see in Table 5 this is not always the case. A majority class classifier can perform better than a given classifier while the classifier has a positive $\kappa$ statistic. The reason is that the distribution of predicted classes (45\%$55 \%$ ) may substantially differ from the distribution of the actual classes $(70 \%-30 \%)$.

Therefore, we propose to use a new measure that indicates when we are doing better than a majority class classifier, and name it $\kappa_{m}$ statistic. The $\kappa_{m}$ statistic is defined as:

$$
\kappa_{m}=\frac{p_{0}-p_{m}}{1-p_{m}} .
$$

The quantity $p_{0}$ is the classifier's prequential accuracy, and $p_{m}$ is the prequential accuracy of a majority class classifier. If the classifier is always correct then $\kappa_{m}=1$. If its predictions are correct as often as those of a majority class classifier, then $\kappa_{m}=0$.

In the example of Table 5 , the majority classifier acquires accuracy of 0.7 , and prequential accuracy is 0.65 , then $\kappa_{m}=$ $\frac{0.65-0.7}{1-0.7}=-16.67 \%$. This negative value of $\kappa_{m}$ shows that the classifier is performing worse than the majority class classifier.

In summary, we propose to use the $\kappa_{m}$ statistic to address issue I3, a measure that is easily comprehensible, with a behavior similar to $C M D$ and $\kappa$, and that deals correctly with the problems introduced by skew in evolving data streams.

\section{$5.4 \kappa_{m}$ Statistic Evaluation}

The main motivation of using the $\kappa_{m}$ statistic is when data streams are evolving class unbalanced. We show that this measure has advantages over accuracy and $\kappa$-statistic: it is a comprehensible measure, and it has a zero value for a majority class classifier.

We perform a prequential evaluation with a sliding window of 1000 examples, on the Electricity dataset, where the class label identifies the change of the price relative to a moving average of electricity demand over the previous 24 hours; with a total of 45,312 examples. This dataset is a widely used dataset described by Harries [15] and analysed

Table 5: Simple confusion matrix example.

\begin{tabular}{lccc}
\hline & $\begin{array}{c}\text { Predicted } \\
\text { Class+ }\end{array}$ & $\begin{array}{c}\text { Predicted } \\
\text { Class- }\end{array}$ & Total \\
\hline Correct Class+ & 40 & 30 & 70 \\
Correct Class- & 5 & 25 & 30 \\
\hline Total & 45 & 55 & 100 \\
\hline
\end{tabular}



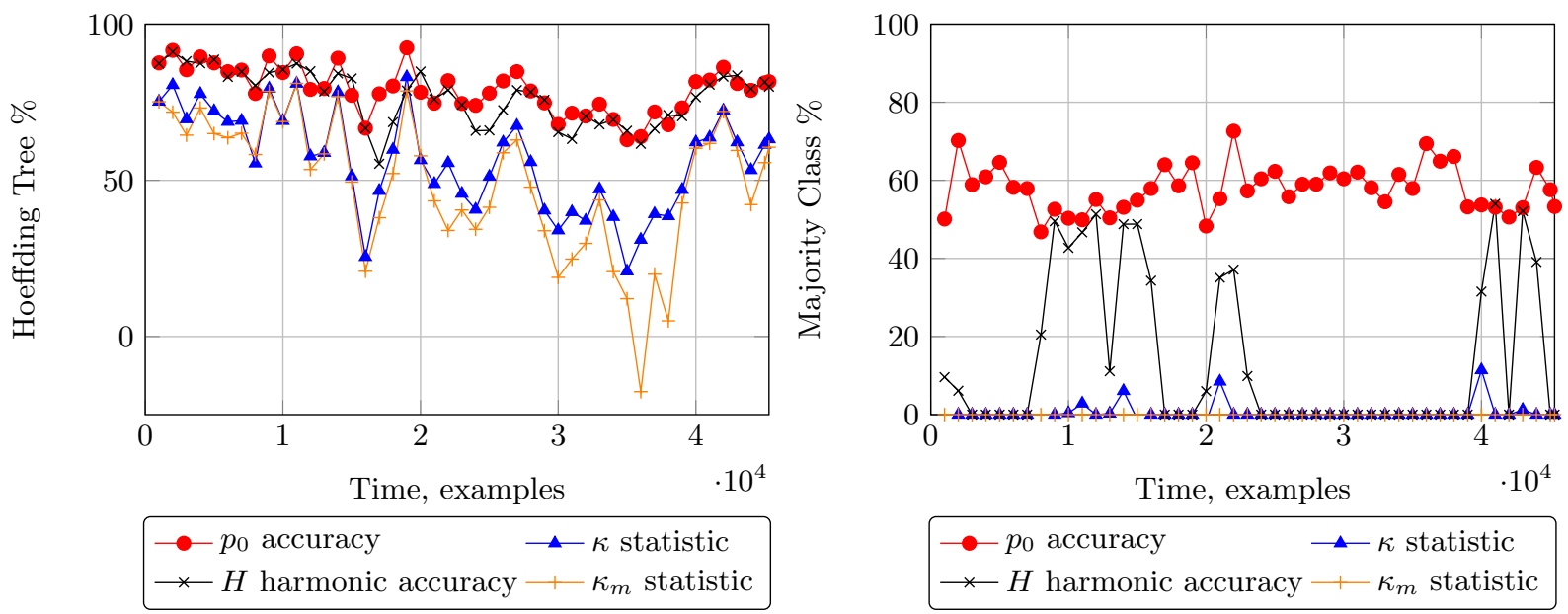

Figure 2: Accuracy, $\kappa$ Statistic, Harmonic Accuracy, and $\kappa_{m}$ Statistic of a Hoeffding Tree (left) and a Majority Class classifier (right) on the Electricity Market Dataset.

by Gama 12. This dataset was collected from the Australian New South Wales Electricity Market. In this market, prices are not fixed but are affected by demand and supply of the market, and are set every five minutes.

Comparing the usage of $p_{0}, \kappa$ statistic, harmonic accuracy $H$ and $\kappa_{m}$ statistic (Figure 2), we see that for the Hoeffding tree, the $\kappa_{m}$ statistic is similar or lower to the $\kappa$ statistic, and for a period of time it is negative. Negative values indicate that the Hoeffding tree is doing worse than a simple majority class classifier. This behaviour is not discovered by the other measures, thus showing the benefit of using this new $\kappa_{m}$ statistic measure.

In the right part of Figure 2, we compare the different measures for the majority class classifier that uses a sliding window of 1000 examples. Of course the $\kappa_{m}$ statistic has a constant value of zero, but the $\kappa$ statistic and harmonic accuracy $H$ have some positive values. This behavior is due to the evolution of the class labels, as at a certain point the majority switches from one label to the other. In this window the $\kappa$ statistic can be positive. Again, we see the benefit of using this new $\kappa_{m}$ statistic measure.

\subsubsection{The Kappa-Temporal Statistic Measure}

Considering the presence of temporal dependencies in data streams, a new measure the Kappa-Temporal statistic was proposed in 27, defined as

$$
\kappa_{p e r}=\frac{p-p_{p e r}}{1-p_{p e r}}
$$

where $p_{p e r}$ is the accuracy of the Persistent classifier. The Persistent classifier is a classifier that predicts that the next class label will be the same as the last seen class label.

We would like to note that $\kappa_{p e r}$ corresponds to the $\kappa_{m}$ measure computed using a sliding window of size 1 . Computing the majority class of a sliding window of size 1 is the same as using the last seen class label.

\section{REAL-TIME PREQUENTIAL MEASURE}

The performance of a classifier may be evolving over time. We propose to evaluate learning systems using real-time measures of performance: the average of the prequential measures in the most recent sliding window containing data that corresponds to the current distribution of data.

Holdout evaluation gives a more accurate estimation of the accuracy of the classifier on more recent data. However, it requires recent test data that it is difficult to obtain for real datasets. Gama et al. 13] propose to use a forgetting mechanism for estimating holdout accuracy by using prequential accuracy: a sliding window of size $w$ with the most recent observations

$$
p_{0}(t)=\frac{1}{w} \sum_{k=t-w+1}^{t} L\left(\hat{y}_{k}, y_{k}\right),
$$

or fading factors that weigh observations using a decay factor $\alpha$. The output of the two mechanisms is very similar (every window of size $w_{0}$ may be approximated by some decay factor $\alpha_{0}$ ). The authors note in their paper that "we observed that the choice of fading factors and the window size is critical."

As the output will depend on the scale of change of the data stream, we propose to use accuracy and $\kappa_{m}$ statistic measured using an adaptive sliding window such as ADWIN [2], an algorithm for estimating mean and variance, detecting change and dynamically adjusting the length of a data window to keep only recent data.

ADWIN keeps a variable-length window of recently seen items, for example loss function values $L\left(\hat{y}_{k}, y_{k}\right)$ in a classification task, such that the window has the maximal length statistically consistent with the hypothesis "there has been no change in the average value inside the window."

More precisely, an older fragment of the window is dropped if and only if there is enough evidence that its average value differs from that of the rest of the window. This has two consequences: one, that change is reliably declared whenever the window shrinks; and two, that at any time the average over the existing window can be reliably taken as an estimation of the current average in the stream.

ADWIN is parameter- and assumption-free in the sense that it automatically detects and adapts to the current rate of change. Its only parameter is a confidence bound $\delta$, indicating the desired confidence in the algorithm's output, inherent to all algorithms dealing with random processes. 
In our case, ADWIN keeps a sliding window $W$ with the most recent $x_{t}=L\left(\hat{y}_{t}, y_{t}\right)$. Let $n$ denote the length of $W$, $W_{0} \cdot W_{1}$ a partition of $W, \hat{\mu}_{W}$ the (observed) average of the elements in $W$, and $\mu_{W}$ the (unknown) average of $\mu_{t}$ for $t \in W$.

Since the values of $\mu_{t}$ can oscillate wildly, there is no guarantee that $\mu_{W}$ or $\hat{\mu}_{W}$ will be anywhere close to the instantaneous value $\mu_{t}$, even for long $W$. However, $\mu_{W}$ is the expected value of $\hat{\mu}_{W}$, so $\mu_{W}$ and $\hat{\mu}_{W}$ do get close as $W$ grows.

Now we state our main technical result about computing prequential accuracy using ADWIN based in [2]:

THEOREM 3. Let $n_{0}$ and $n_{1}$ be the lengths of $W_{0}$ and $W_{1}$ and $n$ be the length of $W$, so that $n=n_{0}+n_{1}$. Let $\hat{\mu}_{W_{0}}$ and $\hat{\mu}_{W_{1}}$ be the averages of the values in $W_{0}$ and $W_{1}$, and $\mu_{W_{0}}$ and $\mu_{W_{1}}$ their expected values. During the evaluation, at every time step we have:

1. (False positive rate bound). If $\mu_{t}$ remains constant within $W$, the probability that ADWIN detects a change and shrinks the window at this step is at most $\delta$.

2. (False negative rate bound). Suppose that for some partition of $W$ in two parts $W_{0} W_{1}$ (where $W_{1}$ contains the most recent items) we have $\left|\mu_{W_{0}}-\mu_{W_{1}}\right|>2 \epsilon_{c u t}$, where

$$
\begin{aligned}
m & \left.=\frac{1}{1 / n_{0}+1 / n_{1}} \text { (harmonic mean of } n_{0} \text { and } n_{1}\right), \\
\delta^{\prime} & =\frac{\delta}{n}, \text { and } \quad \epsilon_{\text {cut }}=\sqrt{\frac{1}{2 m} \cdot \ln \frac{4}{\delta^{\prime}}} .
\end{aligned}
$$

Then with probability $1-\delta$ ADWIN detects a change and shrinks $W$ to $W_{1}$, or shorter.

ADWIN is efficient, since the total processing time per example is $O(\log W)$ (amortized) and $O(\log W)$ (worst-case).

\subsection{ADWIN Prequential Evaluation}

In the next experiment, we plot the prequential evaluation for three different sizes of a sliding window: 100, 1000 , and 10000. The plots are shown in Figure 3. We observe that the plots are different depending on the size of the window. In this example, where we plot the accuracy of a Hoeffding Tree using the Electricity dataset, we see that with a size of 10000 , the plot is smoothed, with a size of 100 , there are many fluctuations, and that the size that is more similar to the ADWIN prequential accuracy is the sliding window of size 1000 . We note that using ADWIN the evaluator does not need to choose a size for the sliding window, and that it has theoretical guarantees that the chosen size is optimal.

Figure 4 shows a comparison between a hold-out evaluation, a prequential evaluation using ADWIN, a sliding window of 100 and a sliding window of 10000 . We observe that the prequential evaluation using ADWIN is very similar to hold-out evaluation, and that short sliding windows have some fluctuations, and large sized sliding windows may have some delay. For evolving data streams with different rates of change, the size of the optimal window is not always the same. ADWIN prequential evaluation is an easy way to have an estimation similar to hold-out evaluation without needing testing data, and without needing to decide the optimal size of a sliding window for evaluation.
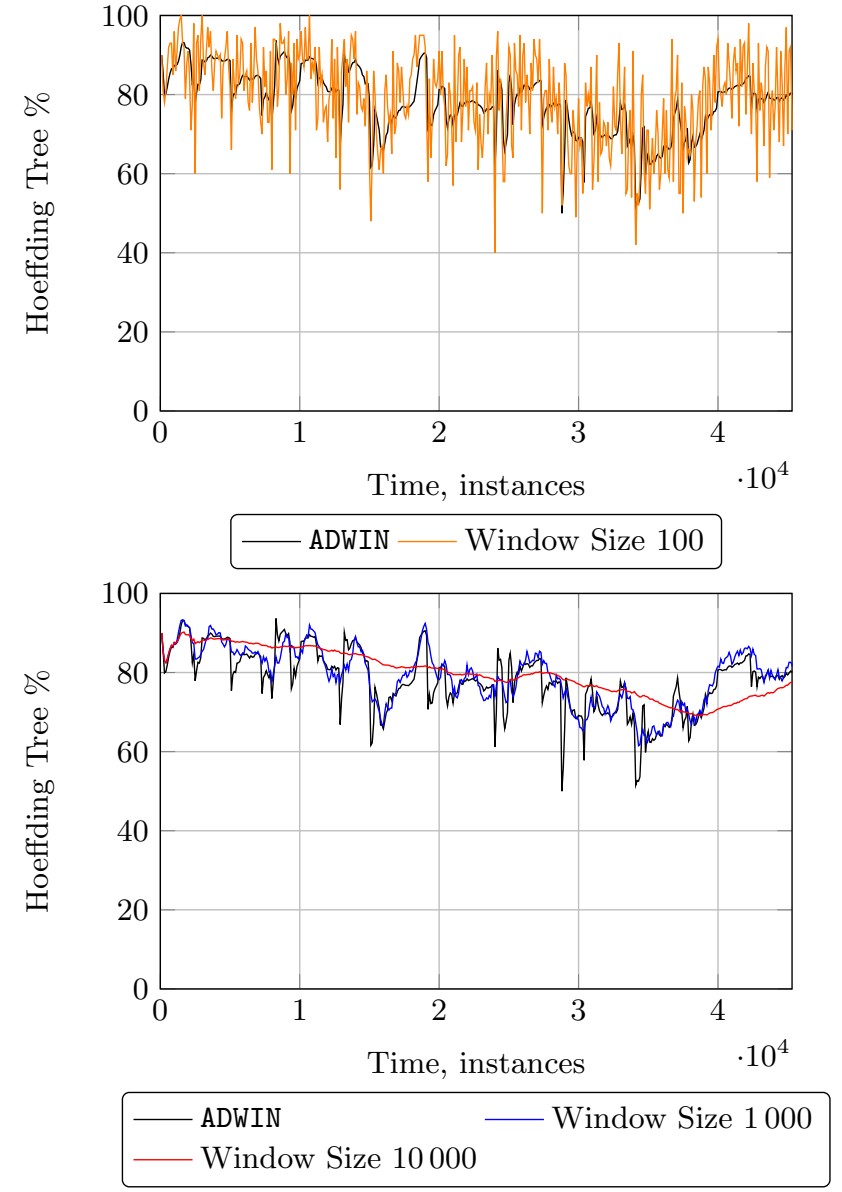

Figure 3: Accuracy using a sliding window of 100, 1000 , 10000 and ADWIN for the Electricity Market Dataset

\section{CONCLUSIONS}

Evaluating data streams online and in a distributed environment opens new challenges. In this paper we discussed three of the most common issues, namely: (I1) which validation procedure to use, (I2) the choice of the right statistical test, (I3) how to deal with unbalanced classes, and (I4) and the proper forgetting mechanism. We gave insights into each of these issues, and proposed several solutions: prequential $k$-fold distributed bootstrap (I1), Wilcoxon's signed-rank test (I2), $\kappa_{m}$ statistic (I3), and ADWIN prequential evaluation (I4). This new evaluation methodology will be available in APACHE SAMOA, a new open-source platform for mining big data streams 9 .

Our main goal was to contribute to the discussion of how distributed streaming classification should be evaluated. As future work, we will extend this methodology to regression, multi-label, and multi-target learning. 


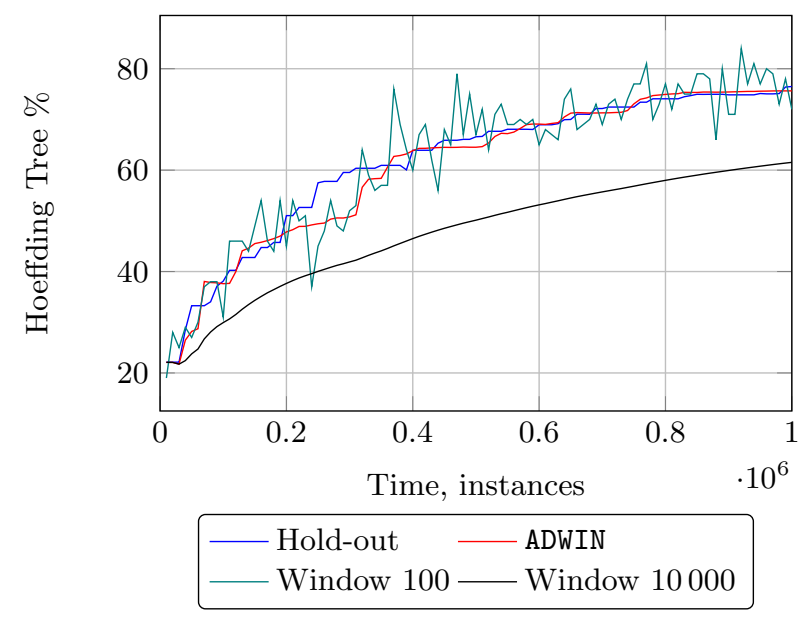

Figure 4: Prequential evaluation with several measures on a stream of a million of instances.

\section{REFERENCES}

[1] A. Asuncion and D.J. Newman. UCI machine learning repository, 2007. URL http://www.ics.uci.edu/ mlearn/MLRepository.html

[2] Albert Bifet and Ricard Gavaldà. Learning from time-changing data with adaptive windowing. In $S D M$, 2007.

[3] Albert Bifet, Geoff Holmes, Richard Kirkby, and Bernhard Pfahringer. MOA: Massive Online Analysis. Journal of Machine Learning Research (JMLR), 2010. URL http://moa.cms.waikato.ac.nz/

[4] Albert Bifet, Geoff Holmes, and Bernhard Pfahringer. Leveraging bagging for evolving data streams. In ECML PKDD, pages 135-150, Berlin, Heidelberg, 2010. Springer-Verlag.

[5] Avrim Blum, Adam Kalai, and John Langford. Beating the hold-out: Bounds for k-fold and progressive cross-validation. In COLT, pages 203-208, 1999.

[6] Remco R. Bouckaert. Choosing between two learning algorithms based on calibrated tests. In $I C M L$, pages 51-58, 2003.

[7] Leo Breiman, J. H. Friedman, R. A. Olshen, and C. J. Stone. Classification and Regression Trees. Wadsworth, 1984.

[8] Jacob Cohen. A coefficient of agreement for nominal scales. Educational and Psychological Measurement, 20(1):37-46, April 1960.

[9] Gianmarco De Francisci Morales and Albert Bifet. SAMOA: Scalable Advanced Massive Online Analysis. Journal of Machine Learning Research, 16:149-153, 2015. URL http://samoa-project.net
[10] Janez Demsar. Statistical comparisons of classifiers over multiple data sets. Journal of Machine Learning Research, 7:1-30, 2006.

[11] Thomas G. Dietterich. Approximate statistical test for comparing supervised classification learning algorithms. Neural Computation, 10(7):1895-1923, 1998.

[12] João Gama, Pedro Medas, Gladys Castillo, and Pedro Pereira Rodrigues. Learning with drift detection. In SBIA, pages 286-295, 2004.

[13] João Gama, Raquel Sebastião, and Pedro Pereira Rodrigues. On evaluating stream learning algorithms. Machine Learning, pages 1-30, 2013.

[14] D. Hand. Classifier technology and the illusion of progress. Statistical Science, 21(1):1-14, 2006.

[15] Michael Harries. Splice-2 comparative evaluation: Electricity pricing. Technical report, The University of South Wales, 1999.

[16] Geoff Hulten and Pedro Domingos. VFML - a toolkit for mining high-speed time-changing data streams. 2003. URL http://www.cs.washington.edu/dm/vfml/.

[17] Geoff Hulten, Laurie Spencer, and Pedro Domingos. Mining time-changing data streams. In $K D D$, pages 97-106, 2001.

[18] N. Japkowicz and M. Shah. Evaluating Learning Algorithms: A Classification Perspective. Cambridge University Press, 2011.

[19] John Langford. Tutorial on practical prediction theory for classification. Journal of Machine Learning Research, 6: 273-306, 2005.

[20] John Langford. Vowpal Wabbit, http://hunch.net/ vw/, 2011. URL http://hunch.net/ vw/

[21] James Manyika, Michael Chui, Brad Brown, Jacques Bughin, Richard Dobbs, Charles Roxburgh, and Angela Hung Byers. Big data: The next frontier for innovation, competition, and productivity. McKinsey Global Institute Report, 2011.

[22] N. Oza and S. Russell. Online bagging and boosting. In Artificial Intelligence and Statistics 2001, pages 105-112. Morgan Kaufmann, 2001.

[23] Nikunj C. Oza and Stuart J. Russell. Experimental comparisons of online and batch versions of bagging and boosting. In $K D D$, pages 359-364, 2001.

[24] Nicos G. Pavlidis, Dimitris K. Tasoulis, Niall M. Adams, and David J. Hand. $\lambda$-Perceptron: An adaptive classifier for data streams. Pattern Recognition, 44(1):78-96, 2011.

[25] Mohak Shah. Generalized agreement statistics over fixed group of experts. In Machine Learning and Knowledge Discovery in Databases - European Conference, ECML PKDD 2011, pages 191-206, 2011.

[26] W. Nick Street and YongSeog Kim. A streaming ensemble algorithm (SEA) for large-scale classification. In $K D D$, pages 377-382, 2001.

[27] Indre Zliobaite, Albert Bifet, Jesse Read, Bernhard Pfahringer, and Geoff Holmes. Evaluation methods and decision theory for classification of streaming data with temporal dependence. Machine Learning, 98(3):455-482, 2015 . 